\title{
Multidisciplinary approach of a locally advanced adult alveolar rhabdomyosarcoma of paranasal sinuses: a case report and literature review*
}

\author{
Maider Campo', Sonia Flamarique', Gemma Asin'1, Ignacio Visus', \\ Alejandra Lacalle², Fernando Mañeru³, Coro Zubimendi', \\ Miguel A. Resano ${ }^{5}$, Javier Saenz ${ }^{6}$, Fernando Arias ${ }^{1}$ \\ 1 Radiation Oncology Department, Complejo Hospitalario de Pamplona Pamplona, Spain \\ ${ }^{2}$ Medical Oncology Department, Complejo Hospitalario de Pamplona Pamplona, Spain \\ ${ }^{3}$ Physics Department, Complejo Hospitalario de Pamplona Pamplona, Spain \\ ${ }^{4}$ Otorhinolaryngology Department, Complejo Hospitalario de Pamplona Pamplona, Spain \\ ${ }_{5}^{5}$ Pathology Department, Complejo Hospitalario de Pamplona Pamplona, Spain \\ ${ }^{6}$ Radiology Department, Complejo Hospitalario de Pamplona Pamplona, Spain
}

Rhinology Online, Vol 1: 104 - 107, 2018

http://doi.org/10.4193/RHINOL/18.034

*Received for publication:

July 18,2018

Accepted: September 11, 2018

Published: September 25, 2018

\begin{abstract}
Alveolar rhabdomyosarcoma (ARMS) is a rare soft-tissue malignancy constituting less than $1 \%$ of soft-tissue sarcomas. In this article we are describing a rare case of ARMS arising in the paranasal sinuses of an adult patient. We emphasize the multidisciplinary treatment administered, thanks to which the patient remains alive and free of disease for six years after the initial diagnosis
\end{abstract}

Key words: paranasal sinuses, paranasal neoplasms, ethmoidal sinus

\section{Introduction}

Alveolar rhabdomyosarcoma (ARMS) is a rare soft-tissues malignancy constituting less than $1 \%$ of soft-tissue sarcomas ${ }^{(1)}$. About $25 \%$ of those occur in the head and neck, where typical sites include orbit, soft tissues of the cheek and paranasal sinuses ${ }^{(2)}$ It primarily affects children and adolescents however it also occasionally occurs in adults.

We describe a rare case of ARMS arising in the paranasal sinuses of an adult patient.

\section{Case report}

A 65-year-old woman was presented to our hospital with a year-long history of oppressive headaches. Her past history was otherwise unremarkable.

Computed tomography (CT) revealed a mass in the ethmoidal air cells and left sphenoidal sinus. The nasal endoscopic examination carried out by our Ear Nose and Throat (ENT) Department showed a mass located in the roof of left nasal fossa. Head and neck physical examination revealed evidence of a palpable, non-mobile, latero-cervical lymph node on the left side. Subsequent T2-weighted magnetic resonance imaging (MRI) showed an aggressive mass in the left ethmoidal sinus (Figure 1). In addition, there were retropharyngeal and upper jugular lymphadenopathies. PET-CT ruled-out distance metastasis. Fine-needle aspiration showed an undifferentiated carcinoma. Microscopic examination of the biopsy specimen revealed a rounded-cell solid tumour, which had grown into solid nest and cords separated by fibrous septa, defining an alveolar pattern. To confirm the diagnosis, FISH analysis was then performed to evaluate for that FKHR gene (13q14) break (Figure 2).

The multidisciplinary tumour board decided to administer 3 cycles of induction chemotherapy, consisting of ifosfamide, doxorrubicine and vincristine, resulting in major response (Figure 3). After that, the patient gave consent for excision of the ethmoidal mass and ipsilateral functional neck dissection. On final pathology analysis, two section margins were reported to contain a residual tumour. To reduce the risk of locorregional recurrence, the patient received adjuvant radiotherapy 60Gy in 30 fractions of 2Gy, using IMRT (Figure 4).

The patient had been recurrence-free for 3 years when in a flexible fibreoptic nasal test we observed a left side protrusion in the nose. MRI showed local tumour recurrence in the left maxillary sinus (Figure 5). An extensive metastatic work-up was negative. With a diagnosis of recurrence ARMS, the patient received a second course of chemotherapy (ifosfamide, vincrsitine, adriamicine, MESNA) resulting in a partial response. The patient was 


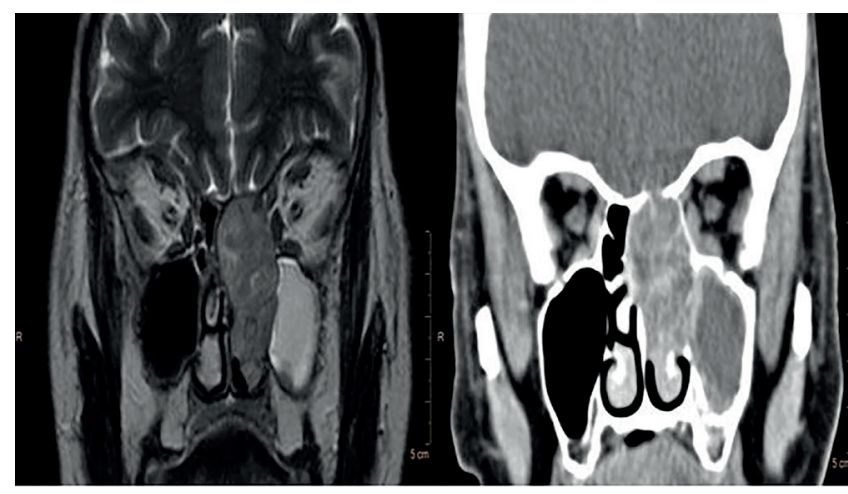

Figure 1. MR-T2 and multi-slice CT with contrast in MPR coronal view. A mass in left ethmoid complex invades nearby structures such as nostril, ipsilateral sphenoid and maxilar sinuses up to skull base.

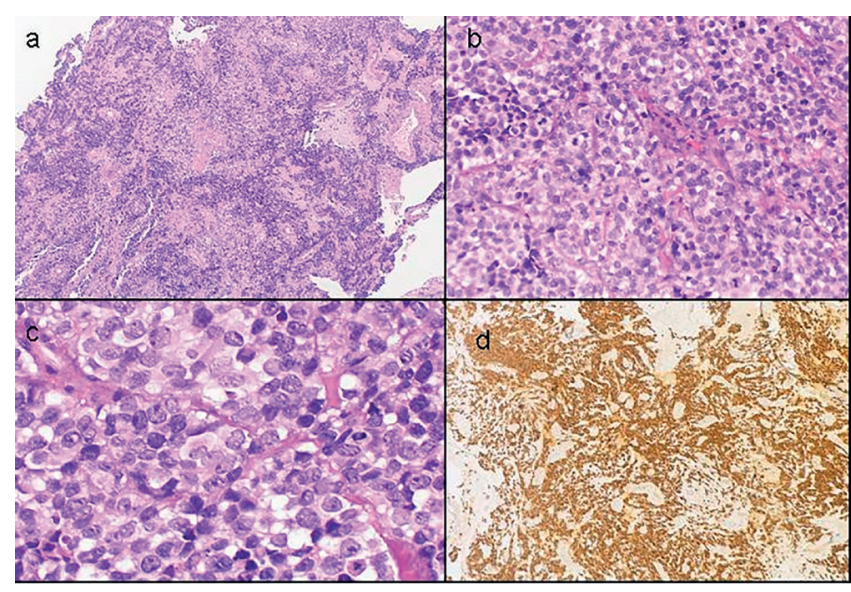

Figure 2. a) Fibrous tissue infiltrated by a small and rounded cell solid tumour, which grows in solid nest and cords separated by fibrous septa, defining an alveolar pattern (H\&E x4). b) Solid nests of rounded and small cells separated by fibrous septa. Some of them have a dark nuclei and some others have a vesiculous nuclei with a slight nucleoli and a clear cytoplasm. There are a few cells with rhabdomyoblastic differentiation. Many mitotic figures are seen $(H \& E \times 20)$ c) Cytologic detail with rounded clear cells with vesiculous nuclei and a slight nucleoli and some others with a dark nuclei and a eosinophilic cytoplasm separated by fibrous septa (H\&E x40). d) Myogenin: positive nuclear stain (x4).

again operated on tumour recurrence. The histological analysis showed the section margin to be positive in maxillary sinus, administrating $60 \mathrm{gy}$ at 2 Gy per fraction over the tumour bed. The patient tolerated the treatment well and now is alive, with a disease-free survival of 33 months after the completion of the second treatment.

\section{Discussion}

Rhabdomyosarcoma (RMS) is a high-grade neoplasm of mesenchymal originates from theprimitive skeletal muscle cells. It is the most common soft tissue sarcoma in childhood and adolescence, but it is extremely rare in adults ${ }^{(3)}$.

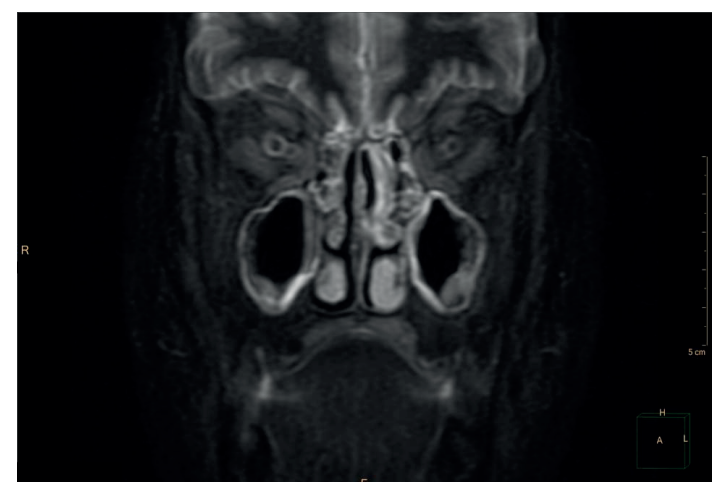

Figure 3. MR- STIR. Rest tumour in left etmoidal bone.

Alveolar RMS is an aggressive subtype with a distinct histology, containing small and rounded cells. Typically, ARMS is rare in the head and neck, and occurs in the deep soft tissues of the lower extremities. Their natural clinical course is indolent and slow usually, presenting functional impairment or as a slowly enlarging mass, as seen in our case ${ }^{(4)}$. Haematogenous spread is the typical route of metastasis, the lung being the most common site in $40-60 \%$ of cases. However, lymphatic metastases are also seen in around $7-10 \%$ of cases.

The diagnosis of ARMS is based on the combination of imaging with very elaborated analyses of the histology, immunochemical and molecular profile. Microscopically, ARMS is characterized by small and rounded cells, containing an abundant clear cytoplasm, with fibrovascular septae separating the tumor cells into nests.

Genetic alterations play an important role in the pathogenesis of the rhabdomyosarcoma. The World Health Organization (WHO) recently revised the classification of RMS subtypes as alveolar rhabdomyosarcoma (ARMS), embryonal rhabdomyosarcoma (ERMS), pleomorphic rhabdomyosarcoma (PRMS), and sclerosing/spindle cell rhabdomyosarcoma (SRMS) in $2013^{(5)}$. The two major histological subtypes of RMS are alveolar RMS, driven by the fusion protein PAX3-FKHR or PAX7-FKHR, and embryonic RMS, which is usually genetically heterogeneous ${ }^{(6)}$.

Effectively, ARMS have a characteristic translocation t $(2 ; 13)$, fusing the PAX3 gene (regulate transcription during neuromuscular development) with the FKHR gene (a member of the family of transcription factors). It is hypothesized that this fusion transcription factor inappropriately activates transcription of the genes that contribute to a transformed phenotype ${ }^{(5)}$. In the same way, the rupture of the FKHR gene has been associated to this histology, as seen in our case.

Because of their extreme rarity, inclusion of the ARMS subtype in the differential diagnosis of small round cell tumors of the head and neck region in patients over the age of 45 years is often neglected.

Due to the rarity of ARMS of the head and neck, having only 


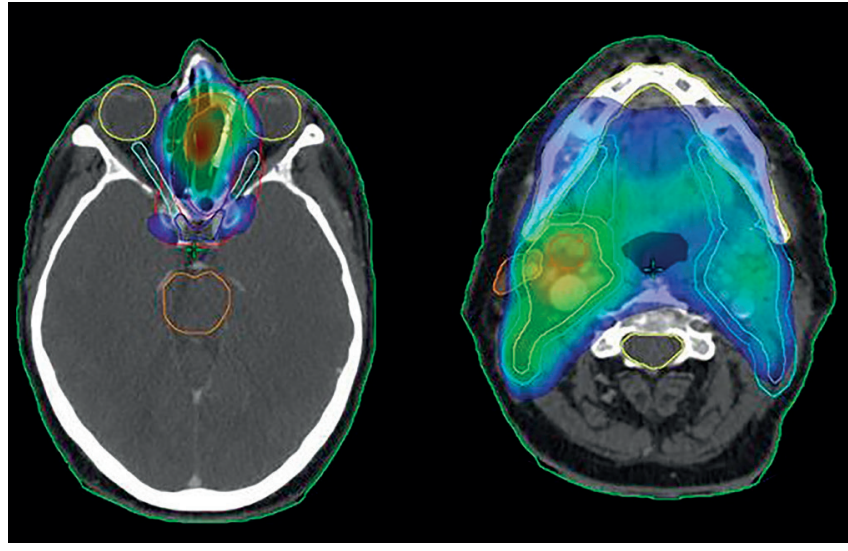

Figure 4. Radiotherapy treatment.

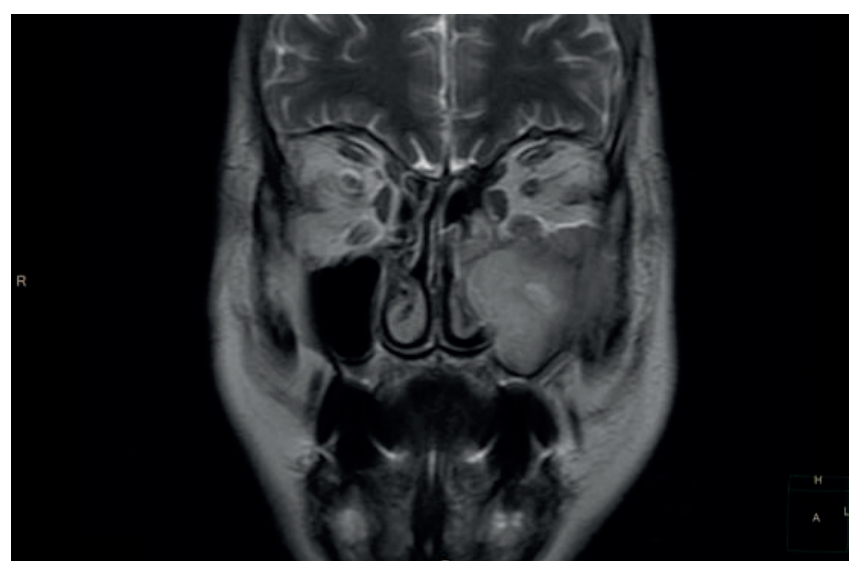

Figure 5. MR-T2 .Tumour recurrence. isolated case reports, the optimal treatment plan has not been clearly elucidated. Multimodality treatment protocols, including surgery, radiotherapy and chemotherapy, have improved the outcome over recent decades ${ }^{(6,7)}$. Local control is the main objective in the treatment of head and neck RMS. Like most soft tissues sarcomas, the main treatment of primary ARMS is complete surgical removal using a wide-local excision. The goal of obtaining negative margins after surgical resection has been shown to increase local control and survival rates. Typically, neck dissection is only utilized when palpable nodes are present, rather than prophylactically. Depending on the tumor location, disease extension and the Center experience, endoscopic surgery can be used ${ }^{(8)}$.

Radiation therapy plays an important role in the treatment of $\mathrm{ARMS}^{(9)}$. It is used to control local microscopic or gross residual disease in such instances, in cases where head and neck localization tumours often cannot be completely removed with surgery. Early guidelines recommended dosage as high as 55 to $60 \mathrm{~Gy}$ for control of the primary tumour. General radiation therapy guidelines have evolved with sequential intergroup studies, concluding that for residual microscopic disease 40-45 Gy appears to be sufficient to achieve local control and 45-50 Gy for gross residual disease.

The development of adjuvant and neoadjuvant chemotherapy has increased survival rates in patients with localized disease to approximately $60 \%$. Combination agents for known activity in the rhabdomyosarcoma include ifosfamide, vincristine, doxorubicine and cyclofosphamida ${ }^{(10,11)}$. The initial approach of our multidisciplinary tumour board was neoadjuvant chemotherapy, due to the unresectability of initial tumour, following surgical excision of the mass and ipsilateral neck dissection. The section margins were affected, so adjuvant radiotherapy was included in the treatment.

Despite treatment improvements, the long-term prognosis for ARMS has remained poor due to the high rate of metastatic disease, being $71 \%$ at five years for patients presenting with localized disease, dropping to $20 \%$ for patients presenting with metastases. The local recurrence rate has been similar, ranging from $10-25 \%$. In our case, our patient obtained 3-years diseasefree with the initial therapy and the same treatments were included in the recurrence, obtaining major response again. Now after 5 years, the patient is alive with no local or distance disease.

The rarity of ARMS in the head and neck region and the smaller clinical series make it difficult to determine prognostic factors for survival.

\section{Conclusion}

In conclusion, our study reports a rare case of ARMS in an infrequent location. Due to the uncommon natural of the disease, diagnosis can be difficult, and analyses of the histopathology and molecular profile features are necessary for confirmation. The optimal treatment for ARMS has not yet been clearly elucidated. A multidisciplinary approach to these patients with surgery, radiotherapy and chemotherapy is the best current therapy, though long-term survivals remains poor.

\section{Authorship contribution}

All the authors have contributed to the draft of the article and accepted the final version.

\section{Conflict of interest}

No known conflict of interest.

\section{References}

1. American Cancer Society. Cancer Facts \& Figures 2014. Atlanta, Ga: American Cancer Society; 2014
2. Turner JH, and. Richmon JD: Head and Neck Rhabdomyosarcoma: A Critical Analysis of Population-Based Incidence and Survival Data. Otolaryngology-Head and
Neck Surgery 2011; 145: 967 - 973

3. Arndt CAS, Crist WM. Common musculoskeletal tumors of childhood and adolescence. N Engl J Med 1999; 341:342-352 
4. Khosla D, Sapkota S, Kapoor R, Kumar R, Sharma SC. Adult rhabdomyosarcoma: Clinical presentation, treatment, and outcome. J Can Res Ther 2015:11: 830-4

5. Fletcher C. D. M., Bridge J. A., Hogendoorn P., Mertens F. WHO Classification of Tumours of Soft Tissue and Bone. 4th. Vol. 5. Paris, France: IARC Press; 2013.

6. Sun X, Guo W, Shen JK, Mankin HJ, Hornicek FJ, Duan Z. Rhabdomyosarcoma: Advances in Molecular and Cellular Biology. Sarcoma. 2015:232010.

7. Barr, FG: Soft tissue tumors: Alveolar rhabdomyosarcoma. Atlas Genet Cytogenet Oncol Haematol. 2009; 13(12):981-985.

8. Gerber NK, Wexler LH, Singer S, et al. Adult Rhabdomyosarcoma Survival Improved with Treatment on Multimodality Protocols.
Int J Radiat Oncol Biol Phys. 2013; 86(1):5863.

9. Crist WM, Anderson JR, Meza JL, et al: rhabdomyosarcoma study-IV: results for patients with nonmetastatic disease. J Clin Oncol. 2001: 19(12): 3091-102.

10. Lund VJ, Wei WI. Endoscopic surgery for malignant sinonasal tumours: an eighteen year experience. Rhinology, 2015; 53(3): 204-11.

11. Eaton, B. R., McDonald, M. W., Kim, S., et al. (2013), Radiation therapy target volume reduction in pediatric rhabdomyosarcoma. Cancer, 119: 1578-1585

12. Esnaola NF, Rubin BP, Baldini EH, et al. Response to Chemotherapy and Predictors of Survival in Adult Rhabdomyosarcoma. Annals of Surgery. 2001; 234 (2):215-223.
13. Ogilvie, C, Crawford, E, Slotcavage, R, et al . Treatment of adult rhabdomyosarcoma. AmJ Clin Oncol 2010, 33: 128-131.

\section{F. Arias}

Department of Radiation Oncology

Complejo Hospitalario de Navarra

Pamplona

Spain

Tel: 0034848422162

E-mail: fariasde@cfnavarra.es

farias.delavega@outlook.es 